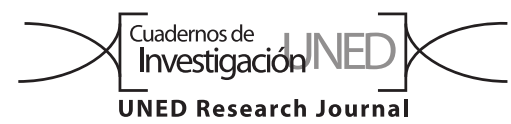

\title{
Bases agroecológicas para la adaptación de la agricultura al cambio climático
}

\author{
Clara Inés Nicholls ${ }^{1} \&$ Miguel A. Altieri ${ }^{2}$ \\ 1. University of California, Berkeley y Sociedad Científica Latino Americana de Agroecología (SOCLA); nicholls@berkeley.edu \\ 2. University of California, Berkeley, USA; agroeco3@berkeley.edu
}

Recibido 30-VII-2018 • Corregido 29-IX-2018 • Aceptado 14-I-2019

\begin{abstract}
Agro-ecological bases for the adaptation of agriculture to climate change. Although many indigenous and peasant populations are particularly exposed to the impacts of climate change and are vulnerable, many communities are actively responding to the changing climate and have demonstrated innovation and resilience, using a variety of strategies to deal with droughts, floods, hurricanes, etc. In this article we argue that traditional agricultural systems offer a wide range of options and management designs that increase the functional biodiversity of crop fields, and therefore, reinforce the resilience of agroecosystems. Many of the traditional agroecological strategies that reduce vulnerability to climatic variability includes the diversification of crops, the maintenance of local genetic diversity, animal integration, the addition of organic matter to the soil, the harvesting of water, etc. Many agroecologists have deciphered the agroecological principles that underlie these strategies which can be adapted taking different technological forms (according to the size of the farms) to design modern agricultural systems so that they become increasingly resilient to the climatic extremes.
\end{abstract}

Key Words: Agroecology, traditional agriculture, adaptation, resilience, climate change.
RESUMEN: Aunque muchas poblaciones indígenas y campesinas están particularmente expuestas a los impactos del cambio climático y son vulnerables, algunas comunidades están activamente respondiendo al clima cambiante y han demostrado innovación y resiliencia, utilizando una diversidad de estrategias para enfrentar las sequías, inundaciones, huracanes, etc. En este artículo argumentamos que los sistemas agrícolas tradicionales ofrecen una amplia gama de opciones y diseños de manejo que incrementan la biodiversidad funcional en los campos de cultivo, y por consiguiente, refuerzan la resiliencia de los agroecosistemas. Muchas de las estrategias agroecológicas tradicionales que reducen la vulnerabilidad a la variabilidad climática incluyen la diversificación de cultivos, el mantenimiento de la diversidad genética local, la integración animal, la adición de materia orgánica al suelo, la cosecha de agua, etc. Varios agroecólogos han descifrado los principios agroecológicos que subyacen a estas estrategias, los cuales se pueden adaptar tomando diferentes formas tecnológicas (de acuerdo al tamaño de las fincas) para diseñar sistemas agrícolas modernos que se tornen cada vez más resilientes a los extremos climáticos.

Palabras claves: Agroecología, agricultura tradicional, resiliencia, adaptación, cambio climático.
La literatura científica y popular reporta que la agricultura es una de las actividades que mas será afectada por el cambio climático, debido a los impactos que las altas temperaturas, sequias y tormentas que se anticipan tendrán sobre la producción vegetal y animal (IPPC, 2014). Generalmente se predice que el cambio climático agravará aún mas las condiciones que experimentan la(o)s campesina(o)s y pequeña(o)s agricultores como resultado de la pobreza, la sensibilidad de sus localidades geográficas (áreas de secano, laderas, etc.) y su alta dependencia en recursos naturales (Jones \& Thornton, 2003). Si bien es cierto que muchas poblaciones indígenas y campesinas están particularmente expuestas a los impactos del cambio climático y son vulnerables, muchas comunidades están activamente respondiendo al clima cambiante y han demostrado innovación y resiliencia, utilizando una diversidad de estrategias para enfrentar las sequías, inundaciones, huracanes, etc. A pesar de estas evidencias, la conclusión prevalente es que la agricultura campesina es particularmente susceptible por su condición de marginalidad y que, aunque los campesinos tengan experiencia en lidiar con la variabilidad climática, sus estrategias tradicionales para enfrentarla no serán suficientes para soportar y resistir la severidad de la variabilidad que se predice. 
Por el otro lado, poco se menciona que la agricultura industrial que ocupa el $70-80 \%$ de los 1500 millones de hectáreas de la tierra arable global (que consume $80 \%$ del petróleo, $80 \%$ del agua y genera entre el $20-30 \%$ de los gases de efecto invernadero) y que solo produce el $30 \%$ de los alimentos para humanos, es la mas susceptible a la variabilidad climática (ETC, 2017). En la totalidad de estas tierras agrícolas se cultiva un puñado de cultivos en forma de monocultivos de gran escala, reduciendo peligrosamente la diversidad genética presente en los sistemas agrícolas globales. Estos sistemas intensivos de monocultivo de impresionante homogeneidad ecológica son particularmente vulnerables al cambio climático, además de a plagas y enfermedades. Este estado ecológico de susceptibilidad en que se encuentra la agricultura industrial constituye una amenaza importante para la seguridad alimentaria de la humanidad (Adams, Ellingboe \& Rossman, 1971).

En este artículo argumentamos que los sistemas agrícolas tradicionales ofrecen una amplia gama de opciones y diseños de manejo que incrementan la biodiversidad funcional en los campos de cultivo, y por consiguiente, refuerzan la resiliencia de los agroecosistemas. Muchas de las estrategias agroecológicas tradicionales que reducen la vulnerabilidad a la variabilidad climática incluyen la diversificación de cultivos, el mantenimiento de la diversidad genética local, la integración animal, la adición de materia orgánica al suelo, la cosecha de agua, etc. Varios agroecólogos han descifrado los principios agroecológicos que subyacen estas estrategias, los cuales se pueden adaptar tomando diferentes formas tecnológicas (de acuerdo al tamaño de las fincas) para diseñar sistemas agrícolas modernos que se tornen cada vez más resilientes a los extremos climáticos.

\section{LA VULNERABILIDAD DE LA AGRICULTURA INDUSTRIAL}

Más de un billón de hectáreas del planeta está dedicada al monocultivo de unos cuantos cereales y animales. Solo el trigo, el maíz, el arroz y la papa representan aproximadamente el $60 \%$ de los alimentos de origen vegetal en el mundo, y solo 14 especies de animales proporcionan el $90 \%$ de todas las proteínas animales. Genéticamente, la agricultura moderna depende asombrosamente de un puñado de variedades para sus principales cultivos (Heinemann, Massaro, Coray, Agapito-Tenfen \& Wen, 2013).

Los datos disponibles indican que en la actualidad la diversidad de los cultivos por unidad de tierra cultivable sigue menguando, lo que en parte se explica por el uso de más de 180 millones de hectáreas de cultivos transgénicos (principalmente soya y maíz) cultivados en todo el mundo y la creciente tendencia a producir grandes monocultivos de maíz, caña de azúcar, palma africana y soya para la elaboración de biocombustibles. Muchos científicos han hecho reiteradas advertencias sobre la vulnerabilidad extrema asociada con la uniformidad genética de los cultivos, afirmando que la homogeneidad ecológica en la agricultura está estrechamente ligada a las invasiones y brotes de plagas y enfermedades.

Estas preocupaciones no son nuevas y quedaron de manifiesto en 1972 con el informe del National Research Council "Genetic Vulnerability of Major Crops" (NRC, 1972) escrito por científicos que alertaron que tragedias tales como la causada por la epidemia de tizón de la hoja de maíz (Helminthosporium maydis) que resultó en una reducción estimada de $15 \%$ en la producción de este cultivo en el medio oeste de Estados Unidos, podría darse también en otros cultivos importantes. Hay muchos otros casos históricos que prueban que la reducción drástica de la diversidad de plantas cultivadas amenaza la producción de alimentos del mundo. La hambruna irlandesa por la destrucción del cultivo de la papa fue el resultado de la diseminación de un clon genéticamente uniforme (de una sola variedad, llamada Lumpers) y el brote de la epidemia del hongo causante del tizón tardío de la papa (Phytophthora infestans), que causó una reducción del $80 \%$ en el rendimiento. Como resultado, millones de irlandeses murieron de hambre y otros dos millones emigraron. La gran hambruna de Bengala en la India en 1943, fue el resultado de una enfermedad devastadora (Cochliobolus miyabeanus) que casi acabó con la producción de arroz. Hace más de un siglo en Francia las cepas de vides fueron totalmente eliminadas por ataques de Phylloxera vertifoliae, hasta que un cultivar resistente fue introducido desde los EEUU (Thrupp, 1998).

Tres décadas más tarde, el tema de la vulnerabilidad agrícola todavía sigue en discusión y el debate continúa sobre el riesgo que representa la homogenización agrícola (hoy en día con la expansión de cultivos transgénicos y biocombustibles) cuando nos enfrentamos al cambio climático (Lobell \& Gourdji, 2012). La susceptibilidad de la agricultura industrial se manifestó durante la peor sequía de los últimos 50 años que afectó severamente la producción de cultivos de los EE.UU en 2012. Se estima que la sequía afectó a 26 de los 52 estados y que cubrió por lo menos $55 \%$ de la superficie terrestre de los EE.UU, es decir casi un billón de hectáreas, con pérdidas económicas sustanciales, y una reducción del rendimiento del 30\%. Después de cuatro años de sequía en California (2011-2015) grandes extensiones de tierra (250 000 hectáreas) permanecieron en barbecho dada la 
falta de agua, lo que representó pérdidas de 1800 millones de dólares y una reducción de 8550 empleos. El reciente huracán Irma que afectó a Florida causó al menos un 30\% de disminución en los rendimientos de muchos cultivos, y los vientos derribaron el 50\% de las frutas de los cítricos, todos producidos en monocultivos.

\section{PROPIEDADES RESILIENTES DE LOS SISTEMAS TRADICIONALES}

Al contrario que los monocultivos de la agricultura industrial, miles de agricultores tradicionales han utilizado sistemas diversificados como policultivos, sistemas agroforestales y silvopastoriles. Existe una asociación positiva entre la diversificación de cultivos y la productividad agrícola, el ingreso de los agricultores, la seguridad alimentaria y la riqueza nutricional.

Estos méritos se deben a la habilidad de los sistemas diversificados de mejorar la fertilidad del suelo, reducir incidencia de plagas y enfermedades, suprimir malezas, y mejorar la eficiencia del sistema, lo que a su vez reduce los riesgos y costos de producción y permite que los agroecosistemas puedan adaptarse a los efectos del cambio climático (Makate, Wang, Makate \& Mango, 2016). Dadas sus características socio-ecológicas descritas en la Figura 1, estos sistemas han permitido a los pequeños agricultores familiares satisfacer sus necesidades de subsistencia en medio de la variabilidad ambiental sin depender de tecnologías agrícolas modernas (Denevan, 1995). Los sistemas agroforestales son ejemplos de sistemas agrícolas con alta complejidad estructural que han demostrado proteger a los cultivos de las grandes fluctuaciones de temperatura manteniéndolos más próximos a sus condiciones óptimas. Por ejemplo, sistemas bajo sombra han demostrado que pueden proteger al café de la disminución de las precipitaciones y la reducción de la disponibilidad de agua del suelo, porque la cobertura arbórea puede reducir la evaporación del suelo y aumentar la infiltración de agua en el suelo (Lin, 2011). Por otra parte, los policultivos anuales permiten a los agricultores producir varios cultivos simultáneamente y minimizar los riesgos (Vandermeer, 1989). Los policultivos manifiestan en general una mayor estabilidad del rendimiento y una menor disminución de la productividad durante las sequías que los monocultivos.

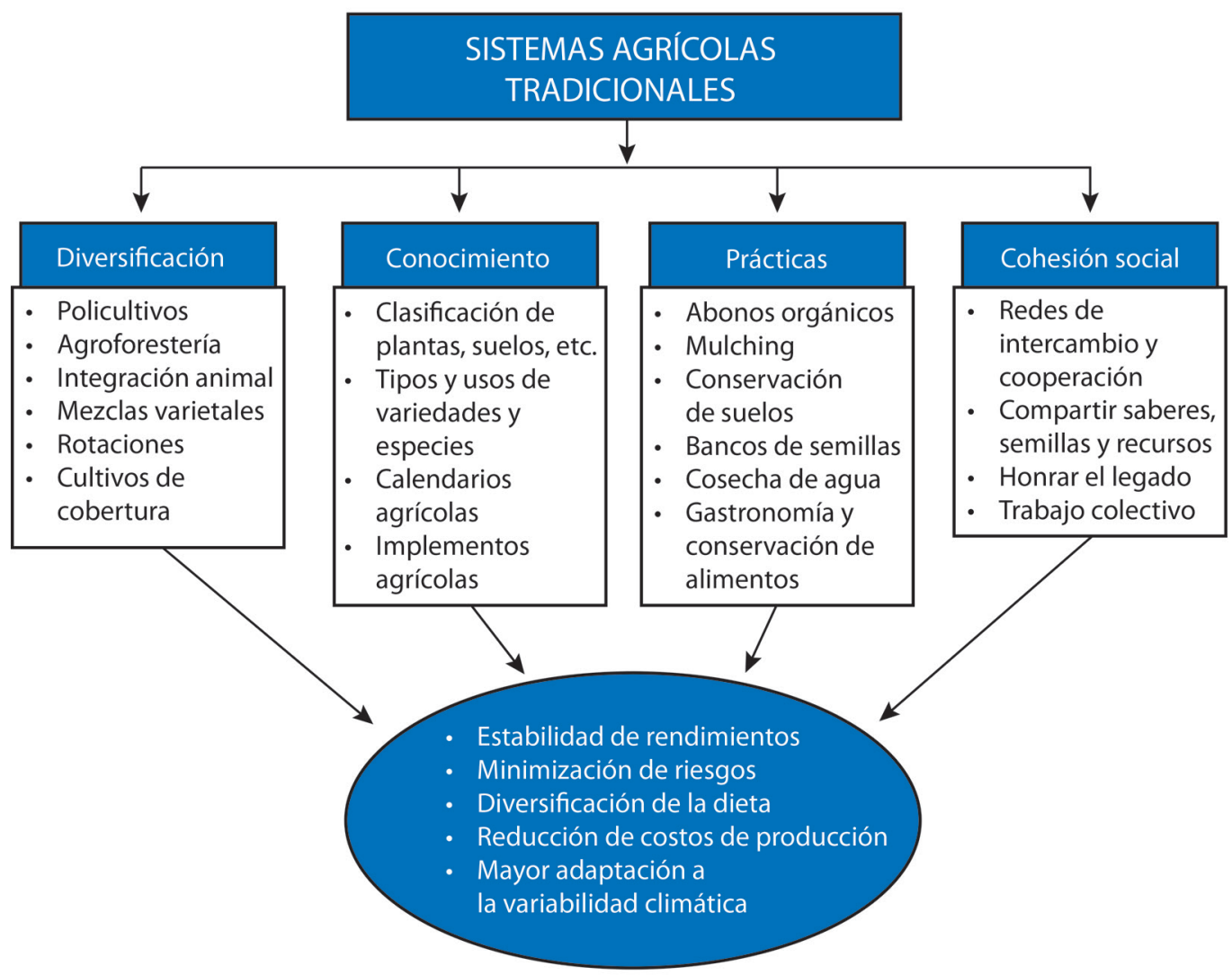

Fig. 1. Características socio-ecológicas de los sistemas agrícolas tradicionales que confieren resiliencia al cambio climático y otros beneficios. 
Los sistemas silvopastorales intensivos (SSI) son una forma sostenible de agrosilvicultura para la producción de ganado que combina arbustos forrajeros sembrados a altas densidades, árboles y palmeras y pastos mejorados. Además de asegurar una gran biomasa forrajera, estos sistemas complejos mantienen un microclima propicio asegurando una alta carga animal y una excelente producción natural de leche y carne (Murgueitio, Calle, Uribea, Calle, \& Solorio, 2011).

\section{CONSTRUYENDO RESILIENCIA EN SISTEMAS MODERNOS}

Durante décadas los agroecólogos han sostenido que una estrategia clave para el diseño de una agricultura sostenible es restaurar la diversidad de las parcelas agrícolas y los paisajes circundantes y manejarla más eficientemente (Altieri \& Nicholls, 2004). La diversificación se propone de muchas maneras: introduciendo variedad genética y aumentando la diversidad de especies como en las mezclas varietales y los policultivos, y en diferentes escalas a nivel de parcelas y paisajes como en el caso de los sistemas silvopastoriles, la integración de cultivos y ganadería, y el enriquecimiento de la matriz paisajística con setos vivos, corredores, etc., proporcionando a los agricultores una amplia variedad de opciones y combinaciones para la implementación de esta estrategia.

Estas prácticas de diversificación deben ir acompañadas con adiciones abundantes de materia orgánica, para crear suelos saludables con una actividad biológica dinámica y buenas características físicas, químicas y biológicas. La materia orgánica es clave para la resiliencia, pues esta aumenta la capacidad de retención de agua del suelo incrementando la tolerancia de los cultivos a las sequías. La materia orgánica también incrementa el nivel de infiltración para disminuir la escorrentía, evitando que las partículas del suelo sean transportadas por el agua durante las lluvias intensas. La materia orgánica mejora la agregación del suelo superficial sosteniendo firmemente las partículas de tierra durante las lluvias o tormentas de viento. Los conglomerados estables resisten el movimiento por el viento o el agua. Los suelos orgánicamente ricos suelen contener altas poblaciones microbianas que influyen en el crecimiento de las plantas y la relación entre plantas, suelo y agua (Magdoff \& Weil, 2004).

\section{Enfrentando las sequias}

Variedades locales: La evidencia demuestra que las variedades locales son menos afectadas por los stress ambientales como la sequía. Al utilizar mejor el agua disponible, las variedades tradicionales generalmente rinden más que las variedades modernas bajo condiciones de stress hídricos. Por ejemplo, en India, variedades locales de trigo exhibieron una producción con respecto al uso de agua tres veces más alta $\left(620,94 \mathrm{~kg} \mathrm{ha}^{-1} \mathrm{~cm}^{-1} \mathrm{de}\right.$ agua) que variedades mejoradas de trigo $\left(293,1 \mathrm{~kg} \mathrm{ha}^{-1}\right.$ $\mathrm{cm}^{-1}$ de agua). Dada esta resistencia, los rendimientos de las variedades locales son más estables que el de las variedades modernas año a año en el mismo campo, o en varios campos en el mismo año. La creación de bancos de semillas comunitarios que recojan el rico germoplasma adaptado a la sequía aun existente en una región, es por lo tanto de un valor estratégico para la adaptación de comunidades a la variabilidad climática (Cleveland, Soleri \& Smith, 1994).

Adición de materia orgánica al suelo: La continua adición de residuos de cosecha, compost y el uso de cultivos de cobertura o abonos verdes incrementan el contenido de materia orgánica, lo que a su vez incrementa la capacidad de almacenamiento de agua del suelo, mejorando la resistencia de cultivos a la sequía. Según el suelo, por cada $1 \%$ de incremento de materia orgánica, el suelo almacena hasta 1,5 litro de agua por metro cuadrado. Investigaciones han mostrado que un incremento de materia orgánica de 0,5\% a 3,0\% duplicó la cantidad de agua disponible para los cultivos (Magdoff \& van Es., 2000).

Activación de la biología del suelo: Un suelo orgánico bien manejado contiene altas poblaciones de bacterias, hongos y actinomicetos. Se han reportado poblaciones bacterianas muy por encima de 5 millones de individuos por gramo de suelo seco, que ayudan a descomponer residuos y a hacer disponible nutrientes. Entre los hongos, la presencia de micorrizas (VAM) que colonizan las raíces de muchos cultivos, es clave ya que estas incrementan la eficiencia del uso del agua lo que ayuda a los cultivos bajo condiciones de stress hídrico (Augé, 2001).

Cobertura del suelo: Manteniendo la vegetación de barbecho sobre el suelo reduce la evaporación conservando en promedio $4 \%$ más agua en el suelo, lo que equivale a unos $8 \mathrm{~mm}$ adicionales de lluvia. Un estudio realizado en Centro América encontró que prácticas agroecológicas como cultivos de cobertura y mulching pueden incrementar el almacenamiento de agua en el suelo entre un 3-15\%. La conservación de agua en el perfil del suelo, hace que los nutrientes estén disponibles de manera inmediata en sincronía con los períodos 
de mayor absorción de los cultivos (Buckles, Triomphe \& Sain, 1998).

Policultivos: Datos de 94 experimentos con varias asociaciones de sorgo con guandul (Cajanus cajan), demostraron que para un "evento extremo" particular, el monocultivo de guandul fallaría una en cinco, el sorgo faIlaría una vez en ocho, mientras que el policultivo fallaba una en 36. Los policultivos exhiben mayor estabilidad en los rendimientos y menores declives de producción que monocultivos bajo condiciones de sequía. Al manipular el estrés hídrico se observó que los cultivos intercalados de sorgo y maní, millo y maní y sorgo y millo rindieron consistentemente más que los monocultivos en cinco niveles de disponibilidad de humedad. Interesantemente, las diferencias relativas en la productividad de los monocultivos y los policultivos se fueron acentuando más conforme aumentaba el estrés (Natarajan \& Willey, 1986). En China, la eficiencia del uso de agua en papas intercaladas con frijoles fue 13,5\% mayor que en monocultivos (Huang et al., 2015).

Sistemas agroforestales: Cuando el café y el cacao se cultivan en sistemas agroforestales, un nivel de sombra de $40-60 \%$ de los arboles crea un microclima que protege a estos cultivos de altas fluctuaciones de temperatura y también de las bajas precipitaciones al reducir la evapotranspiración de agua del suelo. En casos de extrema sequía, muchos agricultores al perder sus cosechas, intercambian madera por alimentos y también suplementan sus dietas con frutas, vainas y hojas de árboles resistentes (Malezieux, 2012).

Sistemas silvopastoriles: Las pasturas enriquecidas con altas densidades de arbustos forrajeros, árboles y palmeras pueden neutralizar los efectos negativos de la sequía. El año 2009 fue el año más seco de los últimos 40 años en el Valle del Cauca- Colombia con una caída en las precipitaciones de 44\%. A pesar de una reducción del $25 \%$ en la biomasa de los pastos, la producción de forraje de árboles y arbustos en la Finca "El Hatico" permitió mantener la producción de leche constante, neutralizando los efectos negativos de la sequía en todo el sistema. Sin embargo, los agricultores de zonas vecinas reportaron pérdidas severas en la producción de leche y en el peso de los animales, además de altas tasas de mortalidad (Murgueitio et al., 2011).

\section{Enfrentando tormentas y huracanes}

En laderas centroamericanas, los agricultores que utilizaban prácticas de diversificación tales como cultivos de cobertura, cultivos intercalados y agroforestería sufrieron menos daños por el huracán Mitch, que sus vecinos que producían monocultivos convencionales. Se encontró que las parcelas diversificadas tenían de $20 \%$ a $40 \%$ más cobertura vegetal, más humedad en el suelo y menos erosión y que experimentaron pérdidas económicas menores que las de sus vecinos convencionales. Los monocultivos de bananos, naranjas y coco son particularmente vulnerables al daño por tormentas, ya que vientos mayores de $64 \mathrm{~km} \mathrm{hr}^{-1}$ pueden causar rompimiento de ramas y dislocación de raíces (Holt-Gimenez, 2002).

En Chiapas, los sistemas de café con sombra diversificada sufrieron menos daños por el huracán Stan que los sistemas de café más simplificados. En zonas afectadas por el huracán Ike en Cuba en 2008, investigadores encontraron que las fincas diversificadas exhibieron pérdidas de productividad del $50 \%$ en comparación con el $90 \%$ o el $100 \%$ en los monocultivos vecinos, a la vez que mostraron una recuperación de la producción más rápida ( $80 \%$ a $90 \%, 40$ días después del huracán) que las fincas de monocultivos (Nicholls, \& Altieri, 2013). Todos estos estudios corroboran que los sistemas agroforestales al incrementar la materia orgánica del suelo mejoran la infiltración del agua, al proveer cobertura previenen la erosión del suelo y muchos árboles actúan como rompe vientos disminuyendo la velocidad del viento y el impacto de las tormentas. Las raíces profundas y superficiales de los árboles también ayudan a estabilizar el suelo (Lin, 2011).

Policultivos de maíz con guandul incrementan la infiltración del suelo (lo que aumenta el agua almacenada en el perfil y reduce la escorrentía) debido a una mayor cobertura del suelo, y mejor estructura del suelo. En suelos manejados con policultivos por 5 años consecutivos, la infiltración se incrementó de $6 \mathrm{~mm} \mathrm{hr}^{-1}$ a $22 \mathrm{~mm} \mathrm{hr}^{-1}$ y experimentaron menos escorrentía (68\%) que en los monocultivos (94\%) (Francis, 1986).

En laderas, cultivos de cobertura como Mucuna pruriens cubren rápido el suelo con mucha biomasa (más de $10 \mathrm{Mg} \mathrm{ha}^{-1}$ ) fijando entre 90-170 $\mathrm{kg} \mathrm{ha}^{-1}$ de $\mathrm{N}$, sobre la cual se siembra maíz alcanzando producciones aceptables de 3,5 a 4,5 $\mathrm{Mg} \mathrm{ha}^{-1}$, evitando erosión, en la ausencia de fertilizantes y sin importar la variabilidad climática. Las prácticas de mulching reducen la exposición del suelo al viento, y además reducen el impacto directo de las 
gotas de lluvias sobre el suelo lo que ayuda a reducir la erosión en laderas (Buckles, Triomphe \& Sain, 1998).

\section{CONCLUSIONES}

La construcción de la resiliencia en sistemas agrícolas consiste primero en entender las características agroecológicas de los sistemas tradicionales y otros sistemas diversificados que han resistido la variabilidad climática y ambiental (Dewalt, 1994). Es clave comprender las ventajas asociadas con la diversificación agrícola que generalmente reduce los riesgos y torna la producción más estable. Los beneficios combinados de los sistemas biodiversificados sobre la regulación del agua, la creación de un microclima favorable, la protección del suelo y el mantenimiento de las reservas de carbono en los sistemas agrícolas diversificados no solo proporciona bienes y servicios ambientales para los productores, sino también una mayor resiliencia al cambio climático (Stigter, Dawei, Onyewotu \& Xurong 2005). En un futuro en el que se pronostica más oscilaciones climáticas dramáticas, introducir una mayor diversidad en los agroecosistemas (como lo hacen los agricultores tradicionales) puede servir de amortiguador frente a los patrones cambiantes de las precipitaciones y la temperatura, y posiblemente permita revertir las tendencias a la baja de los rendimientos a largo plazo conforme una variedad de cultivos y variedades responden de manera diferente a estas perturbaciones (Altieri \& Koohafkan, 2013).

La cuestión a tratar es discernir qué principios y mecanismos han permitido a estos sistemas resistir y/o recuperarse de sequías, tormentas, inundaciones o huracanes. Una vez descifrados los principios que soslayan la resiliencia observada, es posible aplicarlos en el diseño de nuevos sistemas para que sean más resilientes, pero las formas tecnológicas que tomen los principios dependerán del tamaño de las fincas, y las condiciones económicas y ambientales de los agricultores. La red de investigación REDAGRES (www.redagres.org) ha producido una serie de documentos que entregan herramientas metodológicas fáciles para evaluar la resiliencia socio-ecológica de los sistemas agrícolas y así reforzar la capacidad de respuesta de los agricultores (Nicholls \& Altieri, 2013; Henao, Altieri \& Nicholls, 2016).

Dado que el cambio climático ya está ejerciendo sus efectos en la agricultura, un paso urgente es difundir los principios y prácticas de resiliencia utilizados por los agricultores exitosos, así como los resultados de estudios científicos que documentan la efectividad de las prácticas agroecológicas que incrementan la resiliencia de los agroecosistemas a los eventos climáticos extremos (sequías, huracanes, etc.). La difusión eficaz de las tecnologías agroecológicas determinará en gran medida qué tan bien y qué tan rápido puedan adaptarse al cambio climático los agricultores. La difusión a los agricultores de comunidades vecinas y otras en la región puede hacerse por medio de días de campo, visitas recíprocas, seminarios y cursos cortos que expliquen cómo aplicar los principios agroecológicos para mejorar la resistencia tanto a las sequías como a las tormentas fuertes. Quizás la metodología Campesino a Campesino utilizada por miles de agricultores en Mesoamérica y Cuba, que consiste en un mecanismo horizontal de transferencia e intercambio de información, sea la estrategia más viable para difundir las estrategias de adaptación basadas en la agroecología (Holt-Gimenez, 1996).

La capacidad de los grupos o comunidades para adaptarse frente a stress sociales, políticos o ambientales externos debe ir de la mano con la resiliencia ecológica. Para ser resilientes las sociedades rurales deben demostrar capacidad para amortiguar las perturbaciones con métodos agroecológicos adoptados y difundidos a través de la auto-organización y la acción colectiva. El reducir la vulnerabilidad social a través de la ampliación y consolidación de redes sociales, tanto a nivel local como regional, puede contribuir a incrementar la resiliencia de los agroecosistemas. La vulnerabilidad de las comunidades agrícolas depende de lo bien desarrollado que esté su capital natural y social, lo que a su vez hace que los agricultores y sus sistemas sean más o menos vulnerables a las perturbaciones climáticas. En las regiones donde el tejido social se ha roto, el reto será rehabilitar la organización social y las estrategias colectivas en las comunidades, incrementando así la capacidad de respuesta de los agricultores para implementar mecanismos agroecológicos que les permitan resistir y/o recuperarse de los eventos climáticos. El rediseño de los agroecosistemas con principios agroecológicos conlleva a sistemas con propiedades deseables de resiliencia socio-ecológica.

\section{REFERENCIAS}

Adams, M. W., Ellingboe A. H., \& Rossman, E. C. (1971). Biological uniformity and disease epidemics. Bioscience, 21, 10671070. doi:10.2307/ 1295991

Altieri, M. A., \& Nicholls C. I. (2004). Biodiversity and pest management in agroecosystems (2 ed). New York: Haworth Press. DOI:10.2134/ jeq2005.0729

Altieri, M. A., \& Koohafkan, P. (2013). Strengthening resilience of farming systems: A key prerequisite for sustainable agricultural production. In Wake up before it is too late: make agriculture truly sustainable now for food security in a changing climate. UNCTAD, TER13 Report, Geneva. 
Augé, R. M. (2001). Water relations, drought and vesicular-arbuscular mycorrhizal symbiosis', Mycorrhiza, 11, 3-42.

Buckles, D., Triomphe, B., \& Sain, G. (1998). Cover crops in hillside agriculture: farmer innovation with Mucuna. Ottawa, Canada: International Development Research Center.

Cleveland, D. A., Soleri, D., \& Smith, E. A. (1994). Do folk crop varieties have a role in sustainable agriculture? BioScience, $44,740-751$.

Denevan, W. M. (1995). Prehistoric agricultural methods as models for sustainability. Adv Plant Pathol., 11, 21-43. DOI:10.1016/S0736- 4539(06)80004-8

Dewalt, B. R. (1994). Using indigenous knowledge to improve agriculture and natural resource management. Hum Organ., 5, 23-51.

ETC Group. (2017). Who will feed us? The Peasant Food Web vs. the Industrial Food Chain. Retrieved from http://www. etcgroup.org/whowillfeedus

Francis, C .A. (1986). Multiple cropping systems. New York: Macmillan.

Heinemann. J. A., Massaro, M., Coray, D. S., Agapito-Tenfen, S. Z., \& Wen, J. D. (2013). Sustainability and innovation in staple crop production in the US Midwest. International journal of agricultural sustainability, 12(1), 71-88. DOI:10.1080/14735903.2013.806408

Henao, A., Altieri, M. A., \& Nicholls, C. I. (2016). Herramienta didáctica para la planificación de fincas resilientes. Medellín, Colombia: REDAGRES-Instituto Humboldt.

Holt-Gimenez, E. (1996). The Campesino a Campesino movement: farmer- led, sustainable agriculture in Central America and Mexico. In Food First Development Report No 10. Oakland: Institute of Food and Development Policy.

Holt-Giménez, E. (2002). Measuring farmers' agroecological resistance after Hurricane Mitch in Nicaragua: a case study in participatory, sustainable land management impact monitoring. Agriculture, Ecosystems \& Environment, 93(13), 87-105. DOI:10.1016/S0167-8809(02)00006-3

Huang, C., Liu, Q. N., Stomph, T., Li, B., Liu, R., Zhang, H., Wang, C., Li, X., Zhang, C., van der Werf, W., \& Zhang, F. (2015). Economic performance and sustainability of a novel intercropping system on the North China plain', PLOS ONE, 10(8), e0135518.

IPCC. (2014). Climate Change 2014: impacts, adaptation and vulnerability. IPCC Special Report, WGII.

Jones, P. G., \& Thornton, P. K. (2003). The potential impacts of climate change on maize production in Africa and Latin
America in 2055. Global environmental change, 13(1), 51-59.

Lin, B. B. (2011). Resilience in agriculture through crop diversification: adaptive management for environmental change. BioScience, 61, 183-193.

Lobell, D. B., \& Gourdji, S. M. (2012). The influence of climate change on global crop productivity. Plant Physiology, 160, 1686-1697. DOI:10.1104/pp. 112.208298

Magdoff, F., \& van Es., H. (2000). Bulding Soils for Better Crops. Beltsville, M.A.: Sustainable Agriculture Network.

Magdoff, F., \& Weil, R. R. (2004). Soil organic matter management strategies. In Magdoff, F., \& Weil, R.R. (eds.). Soil organic matter in sustainable agriculture (pp. 45-65). Beltsville, M.A.: Sustainable Agriculture Network.

Makate, C., Wang, R., Makate, M., \& Mango, N. (2016). Crop diversification and livelihoods of smallholder farmers in Zimbabwe: adaptive management for environmental change. SpringerPlus, 5(1), 1135. DOI:10.1186/ s40064-016-2802-4

Malezieux, E. (2012). Designing cropping systems from nature. Agronomy for sustainable development, 32(1), 15-29.

Murgueitio, E., Calle, Z., Uribea, F., Calle, A., \& Solorio B. (2011). Native trees and shrubs for the productive rehabilitation of tropical cattle ranching lands. Forest Ecology and Management, 261(10), 1654-1663.

Natarajan, M., \& Willey, R. W. (1986). The effects of water stress on yield advantages of intercropping systems. Field Crops Research, 13, 117-131.

National Research Council, Committee on Genetic Vulnerability of Major Crops. (1972). Genetic vulnerability of major crops. National Academies of Science, Washington.

Nicholls, C. I., \& Altieri, M. A. (2013). Agroecología y cambio climático: metodologías para evaluar la resiliencia socio-ecológica en comunidades rurales. Red Iberoamericana de Agroecología para el desarrollo de sistemas agrícolas resilientes al cambio climático (REDAGRES). Lima, Perú: Gama Grafica.

Stigter, C., Dawei, Z., Onyewotu, L., \& Xurong, M. (2005). Using traditional methods and indigenous technologies for coping with climate variability. Climate Change, 70, 255-271.

Thrupp, L. A. (1988). Cultivating diversity: agrobiodiversity and food security. Washington: World Resources Institute.

Vandermeer, J. (1989). The ecology of intercropping. Cambridge: Cambridge University Press. DOI:10.1017/ CBO9780511623523 